\title{
la gertión del conocimiento como factor de competitividad de las pequeñas y medianas empresas*
}

\section{Knowledge management as a competitiveness factor for small and medium-sized enterprises}

\author{
Josefina Ochoa Ruiz ${ }^{1}$ \\ Amado Olivares Leal ${ }^{2}$ \\ José Coronado Quintana ${ }^{3}$
}

* Derivado de la investigación intitulada Gestión del Conocimiento en las pymes de la ciudad de Hermosillo-México.

1 Universidad de Sonora. jochoa@pitic.uson.mx

2 Universidad de Sonora. olivares@pitic.uson.mx

3 Universidad de Sonora. coronado@pitic.uson.mx 


\section{Gerencia de las organizaciones.}

Un enfoque empresarial

\section{RESUMEN}

El objetivo de esta investigación fue determinar la influencia de la gestión del conocimiento (GC) en la competitividad de las pequeñas y medianas empresas (pymes). Primeramente se realizó una revisión de literatura sobre estos temas y se estudiaron las características propias del escenario de trabajo, las pymes de la ciudad de Hermosillo, Sonora, México. El estudio está soportado en un paradigma cuantitativo, desde un enfoque positivista; de tipo descriptivo, desarrollado bajo un diseño no experimental, de campo, transversal. Tomando en cuenta estas informaciones se elaboró un instrumento de prueba que fue aplicado en una muestra estratificada de la población de estudio. Los datos recabados se procesaron mediante el paquete estadístico PASW Statistics 19. De este análisis se concluyó que las pymes de Hermosillo, presentaron un nivel del 58 \% de uso de las prácticas de la GC, por tanto aún existen muchas áreas de oportunidad en el aprovechamiento de estas prácticas administrativas; también se encontró que los empresarios de las pymes reconocen la importancia e implementación de la gestión del conocimiento en sus empresas como estrategia en la conducción de sus negocios en la búsqueda de una ventaja competitiva, al percibir que influye positivamente para obtener una mayor competitividad en sus empresas.

Palabras clave: competitividad, gestión del conocimiento, pymes.

\section{ABSTRACT}

The objective of this research was to determine the influence of knowledge management (KM) on the competitiveness of small 
and medium enterprises (SMEs). Firstly, a review of the literature on these issues was carried out and the characteristics of the work scenario that was the SMEs of the city of Hermosillo, Sonora, Mexico, were studied. The study is supported in a quantitative paradigm, from a positivist approach; Of descriptive type, developed under a non-experimental, cross-field design, taking into account this information, a test instrument was developed and applied to a stratified sample of the study population. The data collected were processed using the statistical package PASW Statistics 19; Of the analysis of the same was concluded that the SMEs of Hermosillo, presented a level of $58 \%$ of use of the practices of the KM, so there are still many areas of opportunity in the use of these administrative practices; It was also found that SME entrepreneurs recognize the importance of knowledge in their companies and the implementation of management as a strate-

gy in conducting their business in the search for a competitive advantage, perceiving that it influences positively to obtain a Greater competitiveness in their companies.

Keywords: competitiveness, knowledge management, SMEs.

\section{INTRODUCCIÓN}

Ante la creciente dinámica del proceso de creación y difusión del conocimiento, el mundo de los negocios se transforma a gran velocidad en el ámbito económico, financiero, comercial, etc. La competencia nacional e internacional, cada vez más fuerte, obliga a las empresas a ser más competitivas para asegurar su desarrollo en el mejor de los casos o simplemente para garantizar su supervivencia. La formulación de nuevas estrategias de negocios es posible y necesaria, ya que las reglas de la economía 


\section{Gerencia de las organizaciones.}

Un enfoque empresarial

han cambiado en los últimos años; en ese sentido, a diferencia de la economía tradicional, cuyos recursos más valiosos eran sus activos tangibles, en la nueva economía los recursos más valiosos son intangibles.

En este marco, la economía del conocimiento juega un papel muy relevante, y según Drucker (1959), la economía del conocimiento es aquella en la cual la generación y explotación del conocimiento juega un rol predominante en la creación de riqueza; con base en principios como este se sustenta el planteamiento de la relevancia de la administración del conocimiento en las organizaciones.

Las empresas, entre ellas las pequeñas y medianas (pymes), están obligadas a redefinirse de acuerdo a las necesidades de un entorno cambiante, a buscar continuamente todas aquellas prácticas que las lleven a ser más eficientes en su desempeño para mejorar su competitividad tanto nacional como global. Ante esta problemática surgen las siguientes preguntas de investigación: ¿Existe en las empresas pequeñas y medianas una cultura de aprendizaje?, ¿cuál es el nivel de gestión del conocimiento en las pymes?, ¿cuál es la percepción del administrador de empresas de la influencia de la gestión del conocimiento en la competitividad de la firma? Son preguntas que se han formulado diferentes investigadores, gerentes y administradores de empresas cuyas respuestas ayudarían a mejorar su competitividad y desarrollo. El objetivo general de la investigación se centra en determinar la influencia de la gestión del conocimiento en la competitividad de las pequeñas y medianas empresas. El objetivo específico se enfoca en conocer el nivel de gestión del conocimiento que tie- 
nen las empresas pequeñas y medianas localizadas en la ciudad de Hermosillo, Sonora.

\section{ESTADO DEL ARTE Y FUNDAMENTACIÓN TEÓRICA}

Para la realización de este trabajo, primero se revisaron diferentes autores de investigaciones relacionadas con las variables centrales de esta investigación: la gestión del conocimiento y la competitividad de las empresas. Ambas se han analizado en una amplia variedad de estudios profesionales, mismos que han derivado un sinnúmero de conclusiones. Enseguida se hace referencia a algunos autores.

\section{Gestión del conocimiento}

Entre los más notables investigadores de la GC se encuentran Nonaka y Takeuchi (1995), autores del libro Las organizaciones que aprenden, quienes plantean que la gestión del conocimiento se centra en fomentar y facilitar las operaciones que transforman el conocimiento y que dan como resultado la innovación en productos y servicios. Estos autores reconocen la relevancia del conocimiento transformado en nuevos productos y servicios a través de la innovación. Después de Nonaka y Takeuchi, Penrose en Rutihinda (1996), incorpora nuevos elementos al concepto de gestión del conocimiento, reconociéndola como una fuente de ventaja competitiva al afirmar que planteando que lo que distingue una organización de otra, es los recursos especializados que tiene sus activos y habilidades, centrando su valor en la optimización para crear una ventaja competitiva y riqueza económica.

Por su parte, Alavi y Leidner (1999) señalan que la GC corresponden a un proceso sistemático y organizado orientado a la 


\section{Gerencia de las organizaciones.}

Un enfoque empresarial

asquisición, organización y comunicación del conocimiento tácito y explícito por parte de las empresas, para ser mas eficaces y productivos.

Guthrie (2000) incorpora otro elemento esencial al análisis, indicando que la administración del conocimiento es la administración del capital intelectual controlado por la compañía. En ese mismo año, Martensson (2000) señala que la administración del conocimiento es un nuevo camino para entender organización y a las organizaciones, es una herramienta para explotar el conocimiento.

Carlsson (2001) define a la GC aquel proceso donde se identifican, gestionan y utilizan los conocimientos individuales y colectivos para que la empresa sea más competitiva. Shin, Holden

154 y Schmidt (2001) proponen una cadena de valor de la GC, la cual consiste en cuatro actividades básicas: creación del conocimiento, almacenamiento del conocimiento, distribución del conocimiento y aplicación del conocimiento.

El aporte de Jenny Darroch (2003) al análisis, además de que desarrolla un instrumento de prueba para evaluar la gestión del conocimiento en el ámbito microeconómico empresarial, define a este conjunto de prácticas o disciplina administrativa como un proceso que crea conocimiento y administra su transferencia y uso dentro y entre organizaciones. La habilidad de adquirir y utilizar conocimiento, efectivamente es un factor clave en las actividades de innovación y desarrollo de las empresas (Cohen y Levithal, 1990 en Jantunen, 2005).

Hicks, Dattero y Galup (2006) señalan una jerarquía que se inicia 
en el dato, pasa por la información hasta llegar a la gestión del conocimiento. Arbonies (2006) considera que el conocimiento es fundamental para innovar, y recomienda quitar la miopía con la gestión del conocimiento en las organizaciones. Las actividades de la GC incluyen la captura de conocimiento, documentación, recuperación y reutilización; la creación, la transferencia y el intercambio de sus activos de conocimientos integrados en sus procesos operativos y de negocio (Dayan y Evans, 2006).

El estudio de la gestión del conocimiento en las pequeñas y medianas empresas ha motivado investigaciones específicas, ya que presentan diferencias básicas con las grandes organizaciones. Enseguida se presentan algunos resultados obtenidos por diferentes investigadores. La difusión de la GC en la literatura tiende a concentrarse en las grandes empresas (Lee y Kim, 2001; Lin, 2007, 2011). Sin embargo, las diferencias fundamentales entre las grandes empresas y las pymes significan que las conclusiones de los estudios sobre GC en las grandes empresas no pueden aplicarse plenamente a las pymes (McAdam y Reid, 2001; Wong, 2005).

Tan y Lim (2010) sugirieron que los factores críticos de éxito que influyen en los procesos de GC en las pequeñas y medianas empresas (pymes) son la cultura, el liderazgo, la participación de los empleados, de la información y tecnología de las comunicaciones, así como la estructura organizativa. Además, en comparación con las grandes empresas, las pymes en general, tienen estructuras organizacionales planas y flexibles, y procesos elásticos y adaptables, asi como fuerte potencial de innovación (Wong y Aspinwall, 2004). Estas características dotan a las pymes con la flexibilidad organizativa y adaptabilidad que es fundamental para la difusión del éxito de la GC. 


\section{Gerencia de las organizaciones.}

Un enfoque empresarial

Es necesario aumentar las fuerzas competitivas de las pymes y replantearse sus estrategias de competitividad existentes.

De hecho, el conocimiento y su gestión son considerados las más valiosas fuentes de crecimiento y competitividad (Salojarvi, Furu y Sveiby, 2005). Los estudiosos hacen hincapié en que las ventajas de la GC en las pequeñas y medianas empresas, en su mayoría están relacionadas con la reducción de costos, en mejorar la toma de decisiones, en una mayor productividad, en incrementar su participación en el mercado, aumentar la innovación y mejorar su rentabilidad (Lee, Ho y Chiu, 2008; Wang Y.L., Wang, Y.D. y Horng, 2010). Por lo tanto, vale la pena examinar herramientas clave para la difusión de la gestión del conocimiento en las pequeñas y medianas empresas.

\section{Conceptos de competitividad}

La otra variable central de este estudio es la competitividad, misma que ha sido analizada desde distintos enfoques, dando como resultado una amplia variedad de conceptos y metodologías de medición. La competitividad ha sido planteada para los distintos niveles de la economía: a nivel internacional, de país, ciudades y por supuesto, a nivel de industrias y empresas.

El instituto Mexicano para la Competitividad (2010) plantea que la competitividad es la capacidad para atraer y retener inversiones y talento. En sus estudios sobre competitividad en las ciudades, precisa que el objetivo final es lograr que estas no solo sean un lugar donde las personas tengan mejores niveles de vida, sino que estas ciudades sean un punto de atracción para empresas y trabajadores calificados. 
El Instituto Tecnológico de Estudios Superiores de Monterrey (2010), indica que "los pilares de la competitividad deben estar conformados por factores de los cuales depende que los miembros de una sociedad logren niveles de bienestar sustentables, lo cual es el resultado de alcanzar niveles de desarrollo basados en productividad". (p.23)

En indicadores de competitividad para empresas en lo individual, la Organización para el Desarrollo y la Cooperación Económica establece lo siguiente: Los factores que contribuyen a la competitividad microeconómica han sido especial preocupación de la economía administrativa e industrial. Estas disciplinas utilizan una amplia variedad de indicadores (participación en el mercado, beneficios, dividendos, inversión, etc.) para evaluar la competitividad de las empresas.

McFetridge (1995) indica que el concepto de competitividad es mejor entendido en el nivel de la empresa, en los términos más simples; una empresa no rentable es no competitiva. En un modelo de competencia perfecta, una empresa no competitiva es aquella con un costo promedio que excede al precio en el mercado del producto que ofrece, o bien, el valor de los recursos que la empresa está usando excede el valor de los bienes y servicios que está produciendo.

En una industria con producto homogéneo, una firma puede ser no rentable porque su costo promedio es más alto que el costo de sus competidores. Su costo promedio puede ser más alto que el de sus competidores porque su productividad es baja, porque paga más por sus insumos, o ambas. Su productividad puede 


\section{Gerencia de las organizaciones. \\ Un enfoque empresarial}

ser más baja porque es manejada menos eficientemente, opera en unas escalas ineficientes o ambas. Asimismo, en términos de participación en el mercado, una empresa puede mostrar una alta o baja competitividad. En una industria de productos diferenciados, una empresa puede ser no rentable, porque el producto que ofrece es menos atractivo que el de sus competidores.

De esta manera, el nivel de la firma, su rentabilidad, costo, productividad y participación de mercado son todos indicadores de competitividad. El beneficio es un indicador suficiente de competitividad, aunque es mejor medida en un período largo. La participación de mercado puede también ser un indicador suficiente de competitividad si la empresa está maximizando sus beneficios. Por supuesto, una empresa puede ser competitiva en un mercado que está en declive, en cuyo caso, la competitividad no asegura la rentabilidad futura.

Para Porter (1980), en Administración Estratégica, escrito por Thompson y Strickland (2004), la competitividad está en función de cinco fuerzas competitivas: 1) La propia competencia (rivalidad entre vendedores rivales); 2) Los nuevos integrantes potenciales (ingreso potencial de nuevos competidores); 3) Los proveedores (colaboración y negociación entre proveedores y la empresa); 4) Los compradores (colaboración y negociación entre la empresa y los compradores); y 5) Las empresas en otras industrias que ofrecen productos sustitutos (intentos mercadológicos de compañías que ofrecen productos sustitutos para atraer a los clientes de la empresa).

De esta manera una empresa será más competitiva en la medi- 
da que esté mejor preparada para enfrentar a sus competidores actuales con el precio más bajo del mercado, precios más bajos en comparación con el de otros oferentes, productos de mayor calidad o diferenciados, productos de alta diferenciación, o se enfoque en nichos de mercado más especializados y difíciles de atender. Así mismo, una empresa puede lograr mayor competitividad si la industria en la que opera tiene barreras importantes al ingreso de nuevos competidores, ya que disminuye el riesgo potencial de competencia adicional a la ya existente.

Otra fuente de competitividad proviene de la relación entre la empresa y los proveedores, sobre todo si se logra una estrecha relación de cooperación con ellos, tanto que permita tener un poder de negociación importante, en materia de financiamiento, tiempos de pago, disponibilidad de los productos o servicios con la calidad requerida, en momento de entrega necesario, justo a tiempo (just in time), incluso en la secuencia (just in sequence). En el cuarto elemento del modelo de Porter, el poder de negociación entre la empresa y los compradores, la fuente de competitividad proviene de la capacidad que desarrollen la empresas para atender a su mercado, en condiciones que le permitan la sustentabilidad y rentabilidad de sus operaciones.

Adicionalmente, Porter especifica que la competencia para las empresas proviene no solamente de los competidores que ofrecen el mismo producto o servicio, sino de aquellos que ofrecen productos sustitutos, que en un momento dado pueden ser más atractivos a la compra por parte de sus compradores, clientes o consumidores. Este también constituye un factor de competitividad, en el sentido que es necesario desplegar estrategias para 


\section{Gerencia de las organizaciones.}

Un enfoque empresarial

neutralizar los esfuerzos mercadológicos de los productores de bienes sustitutos para superar esta potencial competencia de la empresa.

Existe un acuerdo entre los diferentes autores del tema de competitividad en señalar tres niveles de estudio para esta: el nivel macroeconómico, el sectorial y el empresarial.

En el nivel macroeconómico las variables centrales en materia de competitividad son: los niveles de inversión, las tasas de interés, los gastos en educación, los gastos en inversión y desarrollo, y los tipos de cambio. A nivel sectorial se han identificado las fuerzas competitivas, encontrándose diferencias marcadas en rentabilidad en empresas del mismo sector, por lo que la rentabilidad y ventajas competitivas están más relacionadas con la heteroge-

160 neidad de las empresas en la consolidación de capacidades y en el proceso de construcción de recursos. Aun cuando el estudio de la competitividad se divide en los tres niveles mencionados: macroeconómico, sectorial y microeconómico, lo cierto es que están estrechamente relacionados entre sí.

En ese sentido, como lo indica Porter (1999) en Sastre y Aguilar (2000), para que las empresas - nivel microeconómico-implementen estrategias cada vez más sofisticadas, de administración del conocimiento o cualquier otra, con el propósito de ser más competitivas, son nesesarias mejores infraestructuras, instituciones más avanzadas y personal más calificado - nivel macro-. Este enfoque de competitividad se ha visto complementado, de manera trascendental, por la perspectiva de competitividad sistemática que abona a la comprensión de esta variable y de sus distintos niveles de análisis. 
La tesis central que involucra el concepto de competitividad sistémica, en Meyer-Stamer (2003), indica que las condiciones macroeconómicas y el funcionamiento de los mercados son necesarios, más no suficientes para el desarrollo exitoso de los negocios y de las economías. En ese sentido, es crucial, pero no suficiente, observar los elementos microeconómicos (empresas, consumidores, mercados) y macroeconómicos (tasas de interés, políticas presupuestales y de comercio) cuando se trata de entender porqué los negocios funcionan mejor en un país que en otro; sin embargo, además de los aspectos macro y microeconómicos se observan otros dos agregados.

En el primer agregado están los actores responsables de crear un ambiente favorable de negocios. Este nivel de la competitividad sistémica comprende la interacción de los actores de gobierno y no gobierno, dilucidando si estos actores claves de la economía comparten las orientaciones básicas de los negocios privados, si comparten ideas centrales, como la importancia relativa de los mecanismos de mercado u otros mecanismos de coordinación, así como orientaciones básicas en materia de comercio exterior. Este ámbito de interacción de los principales actores económicos es el primer nivel de análisis de la competitividad sistémica, al cual se le ha denominado Nivel Meta.

Por otro lado, en este esquema de competitividad, entre el nivel macro y micro, los factores importantes para la competitividad de las empresas, pero que no son espontáneamente generados por los mercados, se contemplan en el nivel meso, el cual es el campo de las políticas específicas y los instrumentos de promoción de negocios por parte de las instituciones públi- 


\section{Gerencia de las organizaciones.}

Un enfoque empresarial

cas y privadas involucradas en esta actividad fundamental. La competitividad sistémica significa un patrón de actores, instituciones, organizaciones y prácticas que están interrelacionadas a través de mecanismos complejos, que juntos crean una entidad coherente.

La competitividad sistémica aplicada al nivel local considera diferentes niveles de agregación: supranacional, nacional, regional y local. Como se ha venido planteando, el concepto de competitividad sistémica enfatiza la importancia de los factores determinantes de la evolución del sistema económico, los cuales no son sistemáticamente dirigidos por los enfoques micro y macroeconómicos, distinguiendo entre 4 niveles de agregación: meta, macro, meso y micro e investigando las interrelaciones entre ellos a

162 nivel de las economías nacionales.

Influencia de la gestión del conocimiento en la competitividad de las organizaciones

Como lo señalan Sánchez, Hernández y Haro (2008), los escenarios de competitividad hacen que las empresas planifiquen cómo posicionarse, y a estar preparadas para desarrollar estrategias de supervivencia. En este escenario, tal como lo indican los autores, la estrategia más fuerte de una empresa es desarrollar sus capacidades para crear e innovar en todas las funciones de la organización lo cual solo es posible si implementa y mantiene un sistema de gestión del conocimiento. La habilidad de adquirir y utilizar conocimiento efectivamente es un factor clave en las actividades de innovación y desarrollo de las empresas (Cohen y Levithal, 1990 en Jantunen, 2005). 
Finalmente, algunos autores señalan la influencia positiva entre las prácticas de la gestión del conocimiento y la competitividad de la empresa. Penrose en Rutihinda (1996) reconoce al concepto de gestión del conocimiento como una fuente de ventaja competitiva al afirmar que la competencia distintiva de una firma se basa en los recursos activos y habilidades que posee, centrando su atención en su óptima utilización para construir una ventaja competitiva y riqueza económica. Merali (2000) destaca el carácter estratégico del conocimiento tácito colectivo como el tipo de conocimiento de mayor valor para la empresa; por ser difícilmente articulable y comunicable, difícil de imitar por un competidor.

La práctica de gestión del conocimiento denominada gestión de la creatividad es, de acuerdo a Cabrera y Rincón (2001), la base de la generación de nuevos conocimientos que pueden traducirse en ventajas competitivas, la cual puede aportar valor a la empresa ayudando a crear nuevos productos y servicios, identificando mercados y segmentos de mercado para los productos y servicios existentes, definiendo nuevas formas de elaborar, entregar o comercializar los productos o servicios, y detectando nuevas fuentes de recursos. Por último, como lo señalan Cabrera y Rincón (2001) la cuarta práctica denominada motivación para la gestión del conocimiento, requiere una cultura organizacional orientada en gran medida hacia la colaboración y el intercambio de conocimiento, ideas y experiencias entre las personas que integran las comunidades de conocimiento, grupo de expertos o equipos autodirigidos de la organización, lo cual redunda en beneficios competitivos para las organizaciones. 


\section{Gerencia de las organizaciones.}

Un enfoque empresarial

\section{MÉTODO}

Tomando como base la revisión de los diferentes autores de investigaciones relacionadas con la gestión del conocimiento en organizaciones, así como su influencia en su desempeño, y considerando las características específicas de la población seleccionada, se determinaron las características del presente trabajo: el tipo de investigación, la elaboración del instrumento de prueba, el cual fue sometido a una prueba piloto; después, se definió una muestra representativa del universo, y se realizó un muestreo aleatorio estratificado. Una vez captada la información se procedió a la obtención de resultados utilizando el paquete estadístico PASW Statistics 19; finalmente, se analizaron los resultados obtenidos para llegar a las conclusiones y recomendaciones pertinentes.

\section{Tipo de Investigación}

Esta investigación corresponde a un estudio transversal descriptivo de la percepción del administrador de empresas sobre la influencia de la gestión del conocimiento en la competitividad de las pymes.

\section{Universo de la investigación}

El universo de investigación son las pequeñas y medianas empresas que operan en Hermosillo, inscritas actualmente en el Sistema de Información Empresarial Mexicano (SIEM). Se siguen los criterios de clasificación de empresas según el número de trabajadores y sector indicado por el decreto publicado en el diario oficial de la federación, el 30 de marzo de 1999. En esta clasificación se consideran como pequeñas empresas las que ocupan de 31 a 100 trabajadores en el sector industria, de 6 a 20 en el 
sector comercio y de 21 a 50 para el sector servicios. Para medianas empresas se consideran a las que emplean de 101 a 500 trabajadores en el sector industria, de 21 a 100 si son comercios, y de 51 a 100 para el caso de empresas de servicios.

\section{Elaboración del cuestionario}

Para la elaboración del instrumento de captación de información, se tomaron en cuenta las características especiales de la población estudiada, así como los trabajos de Sánchez et al (2008), Jantunen (2005), Darroch (2003). Con base en esto se consideraron las siguientes variables en cada etapa de la gestión del conocimiento. Para evaluar el grado de gestión del conocimiento se analiza en tres etapas:

- Primera etapa. Filosofía de la organización

- Segunda etapa. Planeación estratégica

- Tercera etapa. Gestión operacional: Procesos clave de gestión del conocimiento

- Identificación del conocimiento,

- Adquisición de conocimiento,

- Desarrollo de conocimiento,

- Diseminación del conocimiento,

- Retención y utilización del conocimiento.

Así mismo se incluyen reactivos para determinar la percepción del administrador de empresas sobre la influencia de la GC en la competitividad de las pymes. Los reactivos incluidos en el instrumento de captación de información fueron valuados considerando la escala de Likert, con cinco posibilidades de respuesta que va de 1: Muy en desacuerdo a 5: Muy de acuerdo. 


\section{Gerencia de las organizaciones.}

Un enfoque empresarial

\section{Muestreo}

Para el tamaño de la muestra se consideró un margen de error del $10 \%$ y un nivel de confianza del $95 \%$, resultando un tamaño de muestra de 120 empresas, de las cuales se seleccionaron 94 pequeñas y 26 medianas.

\section{Recolección de datos}

Los datos fueron recolectados a través de un instrumento de prueba diseñado y evaluado mediante una prueba piloto, para validación de los indicadores utilizados para analizar los factores y variables de estudio. Los cuestionarios fueron aplicados a un total de 120 empresas, lo cual garantiza el $95 \%$ de confiabilidad de los resultados obtenidos a través del muestreo.

\section{Métodos de análisis de datos}

166 Los indicadores fueron analizados utilizando el paquete estadístico IBM SPSS Statistics 19.0, desarrollando un análisis descriptivo de los resultados obtenidos mediante la captación de información con la escala de Likert.

\section{RESULTADOS, DISCUSIÓN Y CONCLUSIÓN}

Enseguida se presentan los resultados obtenidos para cada una de las etapas estudiadas, así como un análisis interpretativo de los mismos.

\section{Primera etapa: filosofía organizacional}

\section{La filosofía organizacional de las pymes de Hermosillo}

En las pymes de Hermosillo, el conocimiento es considerado un elemento valioso para la administración de la organización; con base en lo anterior, el primer aspecto evaluado fue la filosofía organizacional, cuyo resultado indica que 95,4 \% de los empresa- 
rios, gerentes o administradores están "de acuerdo" o "totalmente de acuerdo" (escala de Likert) en que el conocimiento es un factor que influye en gran medida en la forma de administrar la empresa. El empresario pequeño y mediano de la localidad considera en la actualidad a la gestión del conocimiento como una estrategia maestra para dirigir a sus empresas hacia una visión de desarrollo mayor. En congruencia con lo anterior el 93,1 \% de empresarios pymes declaran aplicar la gestión del conocimiento en la búsqueda de una capacidad distintiva o ventaja competitiva para su empresa.

\section{Valores que promueven}

Como se observa en la Tabla 1, las pymes manifiestan que el valor más alto $(94,2 \%)$ es la calidad del producto, mientras que ganar dinero aparece como el objetivo menos prioritario (85,3\%). También se observa que el conocimiento es valorado como un medio para agregar valor a los productos y servicios (93,5 \%). Otro aspecto valorado de manera importante es la creatividad en los empleados y la innovación aplicada a los mismos; así mismo motivan el compartir el conocimiento y el trabajo de equipo. Todos estos resultados pueden aplicarse para generar estrategias que incrementen la competitividad en las pymes de Hermosillo.

\section{Tabla 1}

Valores que los altos mandos de las pymes promueven

\begin{tabular}{lc}
\hline La calidad del producto & $94,2 \%$ \\
$\begin{array}{l}\text { El conocimiento como medio para agregar valor a los productos y } \\
\text { servicios }\end{array}$ & $93,5 \%$ \\
$\begin{array}{l}\text { La creatividad en los empleados y la innovación aplicada a los } \\
\text { productos y servicios }\end{array}$ & $91,8 \%$ \\
\hline Se motiva compartir el conocimiento y el trabajo de equipo & $90,1 \%$ \\
Tener tecnología productiva de punta & $89,5 \%$ \\
\hline Ganar dinero & $85,3 \%$ \\
\hline
\end{tabular}

Fuente: Elaboración propia (2016) 
Gerencia de las organizaciones.

Un enfoque empresarial

\section{Liderazgo}

En cuanto al liderazgo, del tipo que sea, se encontró que el $85 \%$ de las pymes está de acuerdo y muy de acuerdo en que contribuye a la adquisición y desarrollo del conocimiento. El 9 \% permaneció neutral y el 6 \% en desacuerdo. Se observó que en las pequeñas y medianas empresas locales, en un $54 \%$, prevalecen las decisiones por parte del dueño o gerente, de modo que la toma de decisiones se centraliza en la cúspide del organigrama organizacional, ejerciendo un liderazgo clasificado como dictatorial o paternalista. En menor medida (43\%) se observó un liderazgo participativo que incorpora a los distintos niveles jerárquicos en la toma de decisiones, en búsqueda de un nivel mayor de compromiso por parte de los empleados para el logro de las metas trazadas o las decisiones tomadas, de la naturaleza que sean.

\section{8}

\section{Segunda etapa: planeación estratégica}

\section{Planeación estratégica en las pymes de Hermosillo}

Para la planeación estratégica se obtuvieron los resultados mostrados en la siguiente tabla. Se observa que la planeación de largo plazo se ha concretado en planes estratégicos en solo el $78,3 \%$

\section{Tabla 2}

Planeación estratégica en las pymes

\begin{tabular}{|c|c|}
\hline $\begin{array}{l}\text { Los empleados conocen la visión, misión, fortalezas, debilida- } \\
\text { des, amenazas y oportunidades }\end{array}$ & $84,2 \%$ \\
\hline Se cuenta con procedimientos para el desarrollo del trabajo & $83,5 \%$ \\
\hline $\begin{array}{l}\text { La empresa tiene un plan estratégico para su desarrollo y sus } \\
\text { operaciones futuras }\end{array}$ & $78,3 \%$ \\
\hline $\begin{array}{l}\text { Se incluyen objetivos, estrategias y planes de acción para el } \\
\text { desarrollo del conocimiento }\end{array}$ & $77,4 \%$ \\
\hline $\begin{array}{l}\text { Existen funciones y una estructura del personal para el cumpli- } \\
\text { miento de objetivos de gestión del conocimiento }\end{array}$ & $76,8 \%$ \\
\hline
\end{tabular}


el conocimiento estratégico, iniciando con la elaboración de un plan de trabajo a largo plazo que incluya objetivos, estrategias y líneas de acción enfocados a priorizar la adquisición, desarrollo, diseminación y uso del conocimiento estratégico, y enfocarse en gran medida en los procedimientos de trabajo. También es necesaria una estructura de personal dedicado al desarrollo de objetivos y estrategias de gestión del conocimiento, comprometida a dar resultados que le permita a las pymes hermosillenses lograr capacidades distintivas y ventajas competitivas para promover su desarrollo en el largo plazo.

\section{Planeación operativa en las pymes de Hermosillo}

Como se muestra en la Tabla 3, en materia de planeación operativa solo 79,7 \% de las empresas pymes en Hermosillo cuenta con planes de trabajo operativos de corto y mediano plazo, lo cual puede considerarse como otro reto importante en este grupo de empresas de la localidad. Adicionalmente los resultados de la encuesta muestran que no se evalúan los resultados en todas las pymes, ya que solo el 76,4 \% contestó afirmativamente; como es muy difícil mejorar lo que no se mide, casi la cuarta parte de las empresas tiene el reto de evaluar sus resultados en el corto plazo, para estar en posibilidades de obtener mejores resultados. En cuanto a compartir conocimiento al interior de la empresa, se observa que en 88,3 \% de ellas los empleados participan con sus conocimientos, experiencias y creatividad en los procesos de mejora de productos y servicios.

\section{Tabla 3}

\section{Planeación operativa en las pymes}

Los empleados participan con sus conocimientos, experiencias y creatividad en los procesos de mejoras de productos y servicios

Existen planes de trabajo operativo a mediano y corto plazo $88,3 \%$

Se evalúan y miden los logros alcanzados a través de la gestión $79,7 \%$ del conocimiento $76,4 \%$

Fuente: Elaboración propia (2016) 
Gerencia de las organizaciones.

Un enfoque empresarial

\section{Tercera etapa: gestión operacional. Procesos clave de gestión del conocimiento}

En materia de administración o gestión del conocimiento, se analizaron cuatro distintas fases o etapas que lo componen:

1. Identificación de conocimiento,

2. Adquisición y desarrollo,

3. Diseminación,

4. Retención y utilización del conocimiento.

\section{Identificación de conocimiento}

En materia de identificación, 93,8 \% de las empresas conoce las necesidades y preferencias actuales de sus clientes; sin embargo, solo 82,3 \% detecta rápidamente los cambios en el entorno, más específicamente los cambios en las necesidades, gustos y preferencias de sus consumidores. Lo anterior hace reflexionar

170 en la necesidad de trabajar en una mayor flexibilidad de las pymes locales y en su adaptación a los cambios. También es importante el enfoque estratégico con el que se lograría identificar las tendencias de consumo para la innovación de productos y/o servicios que permitieran atender nuevas necesidades en el menor tiempo posible.

\section{Adquisición y desarrollo de conocimiento}

Se observa en la Tabla 4 que aunque en el 81,1 \% de las empresas se promueve la creatividad y la innovación, solo el 54,9 \% realiza inversión en investigación y desarrollo, lo cual atrae la atención, ya que esta es una de las principales fuentes de conocimiento orientado a la innovación. Dado que una de las principales fuentes de conocimiento es la mente de las personas que colabora en una empresa, en las pymes de Hermosillo se observan limitados esfuerzos (58,4 \% de empresas) por aplicar procesos de selección 
de personal con habilidades y aptitudes destacadas en materia de creatividad. Por otra parte, el 76,5 \% señalan a Internet como una fuente de adquisición de ideas, considerándolo como el medio menos costoso; sin embargo, evidentemente es una forma de adquirir conocimiento que tiene una menor calidad e impacto en los procesos de gestión de conocimiento, innovación y competitividad de una organización.

\section{Tabla 4
Adquisición y desarrollo de conocimiento en las pymes de Hermosillo}

\begin{tabular}{ll}
\hline Se promueve la creatividad y la innovación & $81,1 \%$ \\
\hline Se utiliza internet como fuente de ideas & $76,5 \%$ \\
$\begin{array}{l}\text { Se realizan procesos para seleccionar personal creativo } \\
\text { e innovador }\end{array}$ & $58,4 \%$ \\
\hline Se invierte en actividades internas de investigación y desarrollo & $54,9 \%$ \\
\hline
\end{tabular}

Fuente: Elaboración propia (2016)

Tabla 5

Diseminación de conocimiento en las pymes de Hermosillo

\begin{tabular}{lc}
$\begin{array}{l}\text { Se capacita al personal para su puesto de trabajo al ingresar a la } \\
\text { empresa }\end{array}$ & $91,3 \%$ \\
$\begin{array}{l}\text { Frecuentemente se aprovecha el trabajo grupal para compartir } \\
\text { información }\end{array}$ & $80,2 \%$ \\
$\begin{array}{l}\text { Regularmente se realizan reuniones con empleados para com- } \\
\text { partir información }\end{array}$ & $73,8 \%$ \\
$\begin{array}{l}\text { Los empleados son motivados a atender capacitación en semina- } \\
\text { rios y conferencias }\end{array}$ & $63,4 \%$ \\
$\begin{array}{l}\text { Los gerentes frecuentemente dan seminarios y presentaciones a } \\
\text { los grupos de trabajo }\end{array}$ & $46,7 \%$ \\
\hline $\begin{array}{l}\text { Fuente: Elaboración propia } \\
\end{array}$
\end{tabular}

\section{Diseminación de conocimiento}

En la Tabla 5 se manifiesta un mayor esfuerzo de las pymes (91,3 \%) de capacitar a su personal a su ingreso a la empresa. También se observa que un $80,2 \%$ de las pymes aprovecha frecuentemente el trabajo grupal para compartir información de los empleados. Sin embargo, una vez que inician sus actividades, 
Gerencia de las organizaciones.

Un enfoque empresarial

solo el 63,4 \% de las firmas motivan a sus empleados a atender capacitación en seminarios y conferencias; y apenas en el 46,7 \% los gerentes imparten seminarios o presentaciones a sus grupos de trabajo. Las actividades de diseminación se enfocan al trabajo grupal $(82,5 \%)$ y a las reuniones con el personal en el $73,8 \%$ de las firmas.

\section{Retención y utilización del conocimiento}

Como se observa en la Tabla 6, el mayor interés está orientado a los clientes. El 87,9 \% mantiene una base de datos con información actualizada de los clientes a la cual es fácil acceder, y puede ser utilizada para propósito de gestión del conocimiento y aplicación para atender de mejor manera las necesidades, gustos

\section{Tabla 6}

Retención y utilización del conocimiento en las pymes de Hermosillo

\begin{tabular}{lc}
\hline $\begin{array}{l}\text { Se mantiene una base de datos con información actualizada de } \\
\text { los clientes }\end{array}$ & $87,9 \%$ \\
\hline $\begin{array}{l}\text { El desarrollo de productos está acorde con las preferencias y ne- } \\
\text { cesidades de los clientes }\end{array}$ & $82,2 \%$ \\
\hline $\begin{array}{l}\text { Existen registros de mejoras en productos y/o servicios } \\
\text { Se cuenta con información documentada sobre el desarrollo de } \\
\text { productos y/o servicios }\end{array}$ & $78,8 \%$ \\
\hline Patentan nuevos productos ideados por la empresa & $76,4 \%$ \\
\hline Patentan innovaciones de los productos existentes & $33,6 \%$ \\
\hline
\end{tabular}

Fuente: Elaboración rpopia (2016)

y preferencias de los clientes. El 82,2 \% de las pymes considera que el desarrollo de sus productos está en línea con las preferencias y necesidades de los consumidores. Aunque las pymes están reteniendo y utilizando el conocimiento, en este aspecto se observa una gran debilidad, ya que solamente $31,9 \%$ de ellas patentan innovaciones de los productos existentes y apenas 33,6 \% patentan nuevos productos ideados por la empresa, si- 
tuación que puede deberse a dos motivos, el primero, que las empresas no estén realizando innovaciones a sus productos o no estén generando nuevos, y el segundo, es que están generando innovación, pero esta no se está registrando como patente para sus empresas.

\section{Tabla 7}

Distribución porcentual de pymes de Hermosillo según nivel de gestión de conocimiento

\begin{tabular}{lc}
\hline Muy bajo 0-20 & $3,8 \%$ \\
Bajo 21-40 & $11,6 \%$ \\
Medio 41-60 & $36,8 \%$ \\
\hline Alto 61-80 & $38,1 \%$ \\
\hline Muy Alto 81-100 & $9,7 \%$ \\
\hline
\end{tabular}

Fuente: Elaboración propia (2016)

Tabla 8

Nivel de gestión del conocimiento por tamaño de empresa

\begin{tabular}{lc}
\hline \multicolumn{1}{c}{ Tamaño de la empresa } & NGC \\
Total & 58 \\
Pequeña & 57 \\
Mediana & 63 \\
\hline
\end{tabular}

Fuente: Elaboración propia (2016)

\section{Nivel de GC alcanzado por las pymes}

De la información manifestada por las empresas, el nivel de gestión del conocimiento de las pymes de la localidad de Hermosillo, en una escala del 0 al 100 se ubicó en 57 puntos. El nivel de gestión de conocimiento de las pymes hermosillenses es heterogéneo, sin embargo, la proporción de negocios pequeños y medianos posee un grado de gestión del conocimiento medio o superior que prevalece en el entorno empresarial local. En ese sentido, $81,4 \%$ de las firmas pyme de la localidad registra un nivel de gestión de conocimiento medio, alto y muy alto (ver Tabla 7). 
Resultados por tamaño de empresa, del nivel de gestión del conocimiento de las pymes de Hermosillo

En promedio, las pymes registran un nivel de GC de 58. Por tamaño de empresa, las medianas registran un promedio más alto en GC; en ese sentido, mientras las empresas medianas registran un Nivel de GC de 63, las pequeñas, con 6 puntos menos, se ubican en un nivel de 57 (Tabla 8).

Resultados por sector de actividad del nivel de gestión del conocimiento de las pymes de Hermosillo

Por sector de actividad las empresas mejor posicionadas en materia de gestión del conocimiento, como se observa en la Gráfica 10 , son las que pertenecen a la industria, con valor de nivel de GC de 64.

Gráfica 10

Nivel de gestión del conocimiento por sector de actividad

\begin{tabular}{l|c}
\hline \multicolumn{1}{c|}{ Sector de Actividad } & NGC \\
\hline Los tres sectores & 57 \\
Industria & 64 \\
Comercio & 58 \\
Servicios & 56 \\
\hline
\end{tabular}

Fuente: Elaboración propia (2016)

El segundo sector con mejores resultados corresponde al sector comercio, con un nivel de 58, es decir, 6 puntos inferior al sector líder que es el industrial. El sector menos favorecido es el de servicios con un nivel de 56.

Percepción del administrador de empresas sobre la influencia de la GC en la competitividad de las pymes

El administrador de las pymes en Hermosillo, Sonora, manifestó 
la siguiente percepción de la influencia del uso de la GC en sus empresas:

- El 86,3 \% estuvo de acuerdo y muy de acuerdo en que las prácticas de la GC influyeron para que la empresa tuviera una alta participación en el mercado.

- El 79,4 \% estuvo de acuerdo y muy de acuerdo en la influencia de la GC en la rentabilidad del retorno sobre la inversión.

- El 74,7 \% estuvo de acuerdo y muy de acuerdo (Escala Likert) en que el uso de la GC influyó para que la empresa genere utilidades cada año.

Así mismo, el administrador de las pymes percibe que la gestión del conocimiento ha influido en sus firmas para alcanzar los siguientes logros:

- El 87,8 \% de las empresas registran un bajo porcentaje de quejas de sus clientes, y en el 93,8 \% de ellas los clientes se encuentran satisfechos con sus productos y/o servicios.

- En el 59,7 \% de las empresas el precio del producto y/o servicio es el más bajo del mercado, y un 85,4 \% de ellas manifiestan un avance importante sobre su competencia.

En el mundo de negocios actual, el conocimiento se ha venido consolidando como el aspecto más relevante para la innovación, el desarrollo y la competitividad de las organizaciones modernas. La creciente importancia del conocimiento como un nuevo factor de producción trae consigo la necesidad de promover su desarrollo al interior de las organizaciones bajo un esquema metodológico claro y consistente, función que puede realizar efectivamente la gestión del conocimiento. 


\section{Gerencia de las organizaciones.}

Un enfoque empresarial

En esta investigación se elaboró un instrumento de medición que puso de manifiesto el nivel de gestión del conocimiento para las pymes de Hermosillo, Sonora, así como la percepción del administrador de esas empresas sobre la influencia de esas prácticas administrativas en la competitividad de sus firmas.

Las pequeñas y medianas empresas de Hermosillo presentaron un nivel de gestión del conocimiento de 57 en una escala de 0 a 100, por tanto, aún existen muchas áreas de oportunidad en el aprovechamiento de estas prácticas administrativas; sobre todo en el sector primario y en las pequeñas empresas.

Los empresarios de las pequeñas y medianas empresas de Hermosillo reconocen la importancia del conocimiento en sus empresas y de la implementación de la gestión del mismo como una estrategia maestra para la conducción de sus negocios en la búsqueda de una ventaja competitiva al considerar en más del 90 \% que el conocimiento influye en gran medida en la forma de administrar la empresa, que su gestión es una estrategia maestra para dirigir sus negocios a una visión mayor, y en la aplicación de esta estrategia en la búsqueda de una capacidad distintiva o ventaja competitiva.

La planeación estratégica es una práctica administrativa que se aplica en las tres cuartas partes de las empresas, y aún quedan medianas y pequeñas firmas pendientes por implementar y capitalizar sus beneficios a largo plazo. En una proporción similar, este tipo de planes requiere ser reforzado a través de la incorporación de objetivos estratégicos enfocados a la gestión del conocimiento. 
Evidentemente los empleados conocen la visión, misión y valores de la empresa de forma tácita, aun cuando este no se plasme en un plan de manera documentada o explícita, lo cual, sin duda, de llevarse a cabo permitiría reforzar el conocimiento de estos elementos de carácter estratégico.

Asimismo, otro aspecto que predomina es el uso de procedimientos documentados para las operaciones de las empresas, fundamentales en el funcionamiento diario.

En ese mismo sentido, se detectan posibilidades de mejora en la aplicación de prácticas administrativas orientadas a la elaboración de planes de acción con objetivos y estrategias específicas para el desarrollo del conocimiento, y en la contratación de una estructura de personal dedicada específicamente al cumplimiento de los objetivos trazados en materia de gestión del conocimiento.

La formulación y el impulso de una estrategia de esta naturaleza, donde el conocimiento sea la plataforma fundamental sobre la cual se impulse la competitividad empresarial en el largo plazo, requiere de una alta gerencia comprometida con el desarrollo, diseminación, uso y retención del conocimiento que agregue valor; necesita la consolidación de una filosofía organizacional en la cual prevalezcan estos principios y valores.

\section{REFERENCIAS BIBLIOGRÁFICAS}

Alavi, M. y Leidner, D. E. (1999). Knowledge management and knowledge management systems: Issues, challenges, and benefits. Communications of the AIS, 1(7), 1-37. 


\section{Gerencia de las organizaciones.}

Un enfoque empresarial

Arbonies, A. (2006). Conocimiento para innovar: Cómo evitar la miopía en la gestión de conocimiento. Madrid: Ediciones Díaz de Santos.

Cabrera, A. y Rincón, M. (2001). La gestión del conocimiento: Creando competitividad en la nueva economía. México: Nueva Economía y Empresa.

Carlsson, S. A. (2001). Knowledge management in network contexts, in the 9th European Conference on Information Systems. Bled, Slovenia: ECIS Press.

Cohen, M. and Levithal, A. (1990). Absorptive capacity: a new perspective on learning and innovation. Administrative Science Quarterly, 1(35). 128-152.

Darroch, J. (2003). Developing a measure of knowledge management behaviors and practices. Journal of Knowledge Management, 7(5), 41-54.

Dayan, R. y Evans, S. (2006). KM your way to CMMI. Journal of Knowledge Management, 10(1), 69-80.

Diario Oficial de la Federación (1999). Ley para el desarrollo de la competitividad de la micro, pequeña y mediana empresa. México.

Drucker, P. (1959). The Landmarks of Tomorrow. EE.UU: Ediciones Harper.

Guthrie, J. (2000). Intellectual capital review: measurement, reporting and management. Journal of Intellectual Capital, 1(1).

Hicks, R., Dattero, R. y Galup, S. (2006). Los cinco niveles de jerarquía de gestión del conocimiento. Journal of Konowledge Management, 10(1),19-3.

IMCO (2010). Acciones urgentes para las ciudades del futuro: Competitividad Urbana 2010. Cd. México: Instituto Mexicano para la Competitividad. 
ITESM (2010). La competitividad de los Estados Mexicanos 2010. Monterrey: Instituto Tecnológico y de Estudios Superiores de Monterrey.

Jantunen, A. (2005). Knowledge-processing capabilities and innovative performance: an empirical study. European Journal of Innovation Management, 8(3), 336-349.

Lee, C. L., Ho., C. T. y Chiu, Y. L. (2008). The impact of knowledge management enablers on non-financial performance in small and medium enterprises. Journal of Technology Management, 43(1), 266-283.

Lee, J. H. y Kim, Y. G. (2001). A stage model of organizational KM: a latent content analysis. Expert Systems with Applications, 20(4), 299-311.

Lin, H. F. (2007). A stage model of knowledge management: an empirical investigation of process and effectiveness. Journal of Information Science, 33(6) 643-659.

Lin, H. F. (2011). Antecedents of the stage-based knowledge management evolution. Journal of Knowledge Management, 15(1), 136-155.

Martensson, M. (2000). A critical of knowledge management as a management tool. Journal of Knowledge Management, 4(3), 204-216.

McAdam, R. y Reid, R. (2001). SME and large organization perceptions of knowledge management: comparisons and contrasts. Journal of Knowledge Management, 5(3), 231-241.

McFetridge, D. (1995). Competitiveness: Concepts and measures.

Ocasional Paper Number 5. Ottawa: Carleton University Press. Merali, Y. (2000). Individual and Collective Congruence in the Knowledge Management Process. Journal of Strategic Information Systems, (9), 213-234

Meyer-Stamer, J. (2003). Understanding the determinants of Vi- 


\section{Gerencia de las organizaciones.}

Un enfoque empresarial

brant Bussiness Development: The Systematic Competitiveness Perspective. Recuperado de http://www.mesopartner.com Nonaka, I., Takeuchi, H. (1995). The Knowledge-Creating Company. New York: Oxford University Press.

Porter, M. (1980). Competitive Strategy. Thecniques for Analyzing Industries and Competitors. New York: Free Press.

Porter, M. (1999). Microeconomic Competitiveness: Findings from the 1999 Executive Survey. Genova: World Economic Forum Press.

Rutihinda, C. (1996). Resource-based Internalization. Stockholm: Akademitryck AB.

Salojarvi, S., Furu, P. y Sveiby, K. E. (2005). Knowledge management and growth in Finnish SME's, Journal of Knowledge Management, 9(2), 103-122.

Sánchez, J., Hernández, S. y Haro, J. (2008). Un modelo de valoración de la gestión del conocimiento de las empresas. Primera edición. Tepic: Universidad Autónoma de Nayarit.

Sastre, M. y Aguilar, E. (2000). Un modelo de competitividad empresarial basado en recursos humanos. Economía Industrial, 33(2), 125-138.

Shin, M., Holden, T. y Schmidt, R. A. (2001). From knowledge theory to management practice: Towards an integrated approach. Information Processing and Management, 37(2), 335-355.

Tan, C. N. L. y Lim, Y. S.(2010). Knowledge management adoption among Malaysia's SME's: Critical Factors, In the Proceeding of Knowledge Management 5th International Conference 2010. Terengganu: Kuala Terengganu.

Thompson, A. y Strickland, A. J. (2004). Administración Estratégica. México: McGraw-Hill.

Wang, Y. L., Wang, Y. D. y Horng, R. Y. (2010). Learning and inno- 

como factor de competitividad de las pequeñas y medianas empresas

vation in small and medium enterprises. Industrial Management \& Data Systems, 110(2), 175-192.

Wong, K. Y. y Aspinwall, E. (2005). An empirical study of the important factors for knowledge-management adoption in the SME sector. Journal of knowledge Management, 9(3), 64-82.

Wong, K. Y. y Aspinwall, E. (2004). Characterizing knowledge management in the small business environment. Journal of Knowledge Management, 8(3), 44-61. petitividad de las pequeñas y medianas empresas. En Prieto-Pulido, R. y García-Guiliany, J. (2017). Gerencia de las Organizaciones. Un enfoque empresarial. Ediciones Universidad Simón Bolívar. Barranquilla-Colombia. 149-181. 